\title{
Transfer Policies with Discontinuous Lorenz Curves
}

\author{
Johan Fellman \\ Hanken School of Economics, Helsinki, Finland \\ Email: fellman@hanken.fi
}

Received 28 June 2015; accepted 2 February 2016; published 5 February 2016

Copyright (C) 2016 by author and Scientific Research Publishing Inc.

This work is licensed under the Creative Commons Attribution International License (CC BY).

http://creativecommons.org/licenses/by/4.0/

(c) (i) Open Access

\section{Abstract}

In earlier papers, classes of transfer policies have been studied and maximal and minimal Lorenz curves $L(p)$ obtained. In addition, there are policies belonging to the class with given Gini indices or passing through given points in the $(p, L)$ plane. In general, a transformation $h(x)$ describing a realistic transfer policy has to be continuous. In this paper the results are generalized and the class of transfer policies $\{h(x)\}$ is modified so that the members may be discontinuous. If there is an optimal policy which Lorenz dominates all policies in the class, it must be continuous. The necessary and sufficient conditions under which a given differentiable Lorenz curve $\bar{L}(p)$ can be generated by a member of a given class of transfer policies are obtained. These conditions are equivalent to the condition that the transformed variable $Y=h(X)$ stochastically dominates the initial variable $X$. The theory presented is obviously applicable in connection with other income redistributive studies such that the discontinuity can be assumed. If the problem is reductions in taxation, then the reduction for a taxpayer can be considered as a new benefit. The class of transfer policies can also be used for comparisons between different transfer-raising situations.

\section{Keywords}

Lorenz Dominance, Stochastic Dominance, Tax Policy, Transfer Policy

\section{Introduction}

Lorenz curves were initially introduced for comparison and analysis of income distributions in a country in dif-

How to cite this paper: Fellman, J. (2016) Transfer Policies with Discontinuous Lorenz Curves. Journal of Mathematical Finance, 6, 28-33. http://dx.doi.org/10.4236/jmf.2016.61003 
ferent times or in different countries in the same era. Later it has been widely applied in different contexts. Especially, classes of transfer and tax policies have been studied and maximal and minimal Lorenz curves $L(p)$ obtained. In addition, there are policies with given Gini indices or passing through given points in the $(p, L)$ plane. Furthermore, the conditions (stochastic dominance) for attainable Lorenz curves have been obtained ([1], [2]). These findings have been found under the assumption that the transformation is continuous. In this paper we generalize the results for discontinuous transformations.

\section{Notations}

We use similar notations as in my previous papers. Let the income be $X$ with the distribution function $F_{X}(x)$, density function $f_{X}(x)$, mean $\mu_{X}$, and Lorenz curve $L_{X}(p)$. The basic formulae are $\mu_{X}=\int_{0}^{\infty} x f_{X}(x) \mathrm{d} x$ and $L_{X}(p)=\frac{1}{\mu_{X}} \int_{0}^{x_{p}} x f_{X}(x) \mathrm{d} x$, where $F_{X}\left(x_{p}\right)=p$.

We introduce the transformation $Y=h(X)$, where $h(\cdot)$ is non-negative and monotone-increasing. Since the transformation can be considered as a tax or a transfer policy, the transformed variable $Y$ is either the posttax or post-transfer income. The mean and the Lorenz curve for the variable $Y$ are $\mu_{Y}=\int_{0}^{\infty} h(x) f_{X}(x) \mathrm{d} x$ and $L_{Y}(p)=\frac{1}{\mu_{Y}} \int_{0}^{x_{p}} h(x) f_{X}(x) \mathrm{d} x$.

A general theorem concerning Lorenz dominance ([3] [4]) is:

Theorem 1. Let $X$ be an arbitrary non-negative, random variable with the distribution $F_{X}(x)$, mean $\mu_{X}$ and the Lorenz curve $L_{X}(p)$. Let $h(x)$ be a non-negative, monotone-increasing function, let $Y=h(X)$ and let $E(Y)=\mu_{Y}$ exist. The Lorenz curve $L_{Y}(p)$ of $Y$ exists and the following results hold:

1) $L_{Y}(p) \geq L_{X}(p)$ if and only if $\frac{h(x)}{x}$ is monotone-decreasing;

2) $L_{Y}(p)=L_{X}(p)$ if and only if $\frac{h(x)}{x}$ is constant;

3) $L_{Y}(p) \leq L_{X}(p)$ if and only if $\frac{h(x)}{x}$ is monotone-increasing.

\section{Results}

Classes of transfer policies. The class of transfer policies

$$
\boldsymbol{H}:\left\{\begin{array}{l}
h(x) \geq x \\
E(h(X))=\mu_{X}+\rho
\end{array}\right.
$$

where $h(x)$ is non-negative, monotone-increasing and continuous was introduced in ([5] [6]). This class was defined in order to compare policies yielding the same transfer effect. Now we modify this class of transfer policies and allow $h(x)$ to be discontinuous. Define

$$
\boldsymbol{H}^{*}:\left\{\begin{array}{l}
h(x) \geq x \\
E(h(X))=\mu_{X}+\rho
\end{array}\right.
$$

where $h(x)$ is non-negative and monotone-increasing. If $h(x)$ is discontinuous, it can have only a countable number of positive finite steps ([4] [6]). A discontinuous transformation $h(x)$ is sketched in Figure 1.

If an optimal policy exists which Lorenz dominates all policies in $\boldsymbol{H}^{*}$, then according to Theorem 1, it must be continuous because $\frac{h(x)}{x}$ has to be monotonically and decreasing, but of every discontinuity point the ratio 


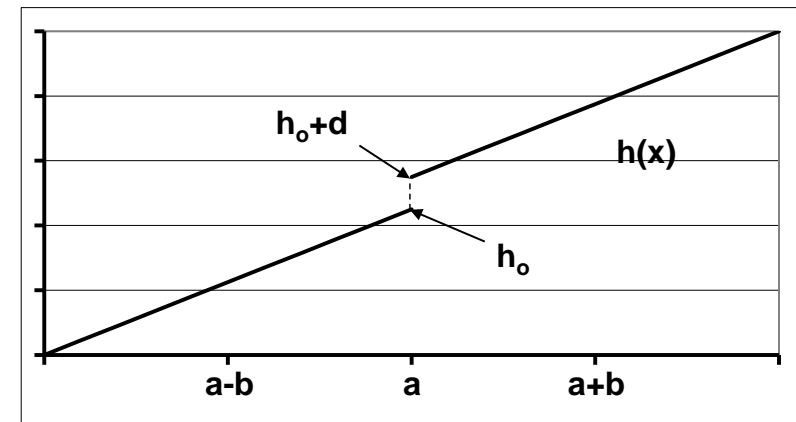

Figure 1. A sketch of a transformation $h(x)$ with a finite pos-

itive jump within the interval $(a-b, a+b)$ (c.f. [4], Figure 1).

$\frac{h(x)}{x}$ cannot be monotonically decreasing. The ratio $v(x)=\frac{h(x)}{x}-\frac{\mu_{Y}}{\mu_{X}}$ is outlined in Figure 2.

Consequently, although class (2), also contains discontinuous policies in comparison with initial class $\boldsymbol{H}$, the policy

$$
h_{0}(x)= \begin{cases}b_{0} & x \leq b_{0} \\ x & x>b_{0}\end{cases}
$$

being optimal among all continuous policies, is still optimal, having the Lorenz curve

$$
L_{0}(p)= \begin{cases}\frac{b_{0}}{\mu_{Y}} p & p \leq q=F_{X}\left(b_{0}\right), \\ \frac{b_{0}}{\mu_{Y}} q+\frac{\mu_{X}}{\mu_{Y}}\left(L_{X}(p)-L_{X}(q)\right) & p>q=F_{X}\left(b_{0}\right) .\end{cases}
$$

The inferior Lorenz curve can be obtained from the sequence [7]

$$
\boldsymbol{H}_{s}: h_{i}(x)=\left\{\begin{array}{ll}
x & x<x_{i} \\
x+k_{i}\left(x-x_{i}\right) & x \geq x_{i}
\end{array} \quad i=1,2, \cdots\right.
$$

These policies give no benefits to the poorest sector of the population $\left(x<x_{i}\right)$, but positive benefits to the richest $\left(x \geq x_{i}\right.$ ). We construct the sequence so that $\boldsymbol{H}_{S} \subseteq \boldsymbol{H}^{*}$ and that their Lorenz curves converge towards an inferior Lorenz curve. If we define $k_{i}(>0)$ so that $\int_{x_{i}}^{\infty} k_{i}\left(x-x_{i}\right) f_{X}(x) \mathrm{d} x=\rho$, then every $h_{i}(x)$ is continuous and monotone increasing: $h_{i}(x) \geq x$ and $E\left(h_{i}(X)\right)=\mu_{X}+\rho$. Hence, $\boldsymbol{H}_{\boldsymbol{S}} \subseteq \boldsymbol{H}^{*}$ and the corresponding Lorenz curve is

$$
L_{i}(p)= \begin{cases}\frac{\mu_{X}}{\mu_{X}+\rho} L_{X}(p) & p<q_{i} \\ \frac{\mu_{X}}{\mu_{X}+\rho} L_{X}\left(q_{i}\right)+\frac{\rho}{\mu_{X}+\rho} \frac{\mu_{X}\left(L_{X}(p)-L_{X}\left(q_{i}\right)\right)-x_{i}\left(p-q_{i}\right)}{\mu_{X}\left(1-L_{X}\left(q_{i}\right)\right)-x_{i}\left(1-q_{i}\right)} & p \geq q_{i}\end{cases}
$$

where $F_{X}\left(x_{i}\right)=q_{i}([7][8])$.

Assume that $k_{i}, x_{i}$ and $q_{i}$ are chosen so that $E\left(h_{i}(X)\right)=\mu_{X}+\rho$ for all $i=1,2,3, \cdots$. Consider a sequence $i=1,2,3, \cdots$, such that $x_{i} \rightarrow \infty, q_{i} \rightarrow 1$ and hence, $k_{i} \rightarrow \infty$. We obtain the limit Lorenz curve [7]

$$
L_{\infty}(p)= \begin{cases}\frac{\mu_{X}}{\mu_{X}+\rho} L_{X}(p) & p<1, \\ 1 & p=1 .\end{cases}
$$




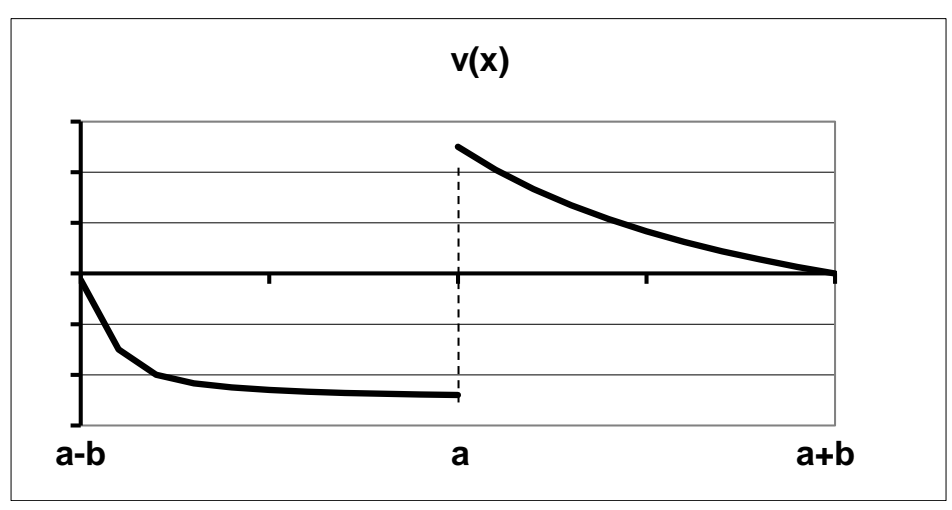

Figure 2. A sketch of the function $v(x)=\frac{h(x)}{x}-\frac{\mu_{Y}}{\mu_{X}}$ within the interval $(a-b, a+b)$ (c.f. [4], Figure 3).

The Lorenz curve is inferior because we can prove [8].

Theorem 2. The Lorenz curve $L_{\infty}(p)$ is inferior to the Lorenz curves for the whole class $\boldsymbol{H}^{*}$.

Proof. Consider an arbitrary, continuous or discontinuous policy $h(x)$ in $\boldsymbol{H}^{*}$. Using the condition $h(x) \geq x$, we can evaluate $L_{\infty}(p)$ in the following way:

$$
L_{h}(p)=\frac{1}{\mu_{X}+\rho} \int_{0}^{x_{p}} h(x) f_{X}(x) \mathrm{d} x \geq \frac{1}{\mu_{X}+\rho} \int_{0}^{x_{p}} x f_{X}(x) \mathrm{d} x=\frac{\mu_{X}}{\mu_{X}+\rho} L_{X}(p)=L_{\infty}(p) .
$$

This inequality holds for all $0 \leq p \leq 1$. Consequently, the class $\boldsymbol{H}^{*}$ of transfer policies containing discontinuous policies satisfies the same properties as the initial class discussed in [5] and [6]. Figure 3 includes a Lorenz curve with a cusp and the Lorenz curves $L_{0}(p)$ and $L_{\infty}(p)$.

A policy with a given Lorenz curve. In Fellman [6] we obtained necessary and sufficient conditions under which a given differentiable Lorenz curve $\bar{L}(p)$ can be generated by a member of a given class of transfer policies. These conditions are equivalent to the condition by which the transformed variable $Y=h(X)$ stochastically dominates the initial variable $X$.

Now we generalise the results, for discontinuous transformations as well. We have stressed above that $h(x)$ can only have a countable number of positive finite steps and that every jump in the transformation $h(x)$ results in a cusp in the Lorenz curve.

One has to assume that the Lorenz curve $\bar{L}(p)$ considered is convex and that it is differentiable everywhere with the exception of a countable number of cusps. The corresponding distribution $\bar{F}_{Y}(y)=M\left(\frac{y}{\mu}\right)$, in which $M(\cdot)$ is the inverse function to $\bar{L}^{\prime}(p)$, with the mean $\mu=\mu_{X}+\rho$ [5]. If $\bar{L}(p)$ has a cusp, then the derivative $\bar{L}^{\prime}(p)$ and the function $M(\cdot)$ have jumps. The cumulative distribution functions are outlined in Figure 4.

In general, when the Lorenz curve $\bar{L}(p)$ and the mean are given, the corresponding income distribution is unique. Now we will prove that the conditions already obtained for classes of continuous transformations still hold for class $\boldsymbol{H}^{*}$; that is, we will characterise attainable Lorenz curves, although they are not universally differentiable.

The crucial part of this proof is to show that $y_{p} \geq x_{p}$ still holds for the distribution $\bar{F}_{Y}(y)$ [6]. The class $\boldsymbol{H}^{*}$ of transfer policies containing discontinuous policies satisfies the same properties as the initial class discussed in [5] and [6]. Following [6], we obtain the transformation $y=\bar{h}(x)=\left(\mu_{X}+\rho\right) \bar{L}^{\prime}\left(F_{X}(x)\right)$. If $\bar{L}(p)$ has a cusp for $p=q$, then $\bar{h}(x)$ has a jump for $x_{q}$. The proof in [6] can be applied as such to $\bar{h}(x)$ whenever it is continuous but the discontinuous points need special attention. Consider a neighbourhood

$x_{q}-b<x<x_{q}+b$, where $x_{q}$ is the only discontinuity point of $\bar{h}(x)$ in the interval $x_{q}-b<x<x_{q}+b$ and choose a $\delta>0$ so small that $x_{q}-b<x_{q-\delta}<x_{q+\delta}<x_{q}+b$. Let $\lim _{\delta \rightarrow 0+} h\left(x_{q-\delta}\right)=y_{q_{-}^{-}}$and $\lim _{\delta \rightarrow 0+} h\left(x_{q+\delta}\right)=y_{q_{+}}>y_{q_{-}}$. 


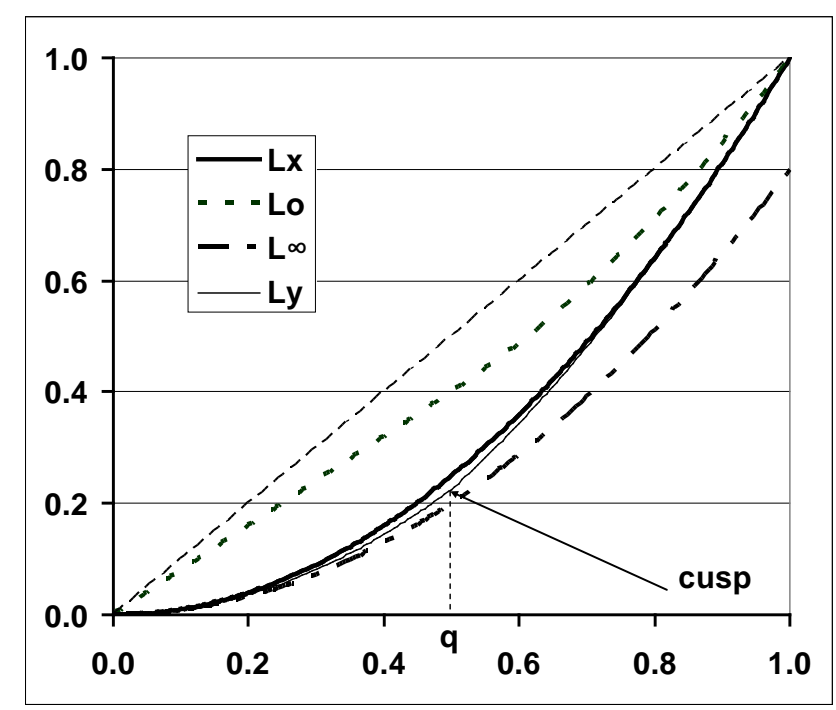

Figure 3. A sketch of the Lorenz curves $L_{X}(p)$ and $L_{Y}(p)$, when $h(x)$ is discontinuous for $x=a$ and $q=F_{X}(a)$. Note the cusp of $L_{Y}(p)$ at the point $p=q$. The figure also includes the maximum and minimum Lorenz curves $L_{0}(p)$ and $L_{\infty}(p)$ for the transfer policies in $H^{*}$.

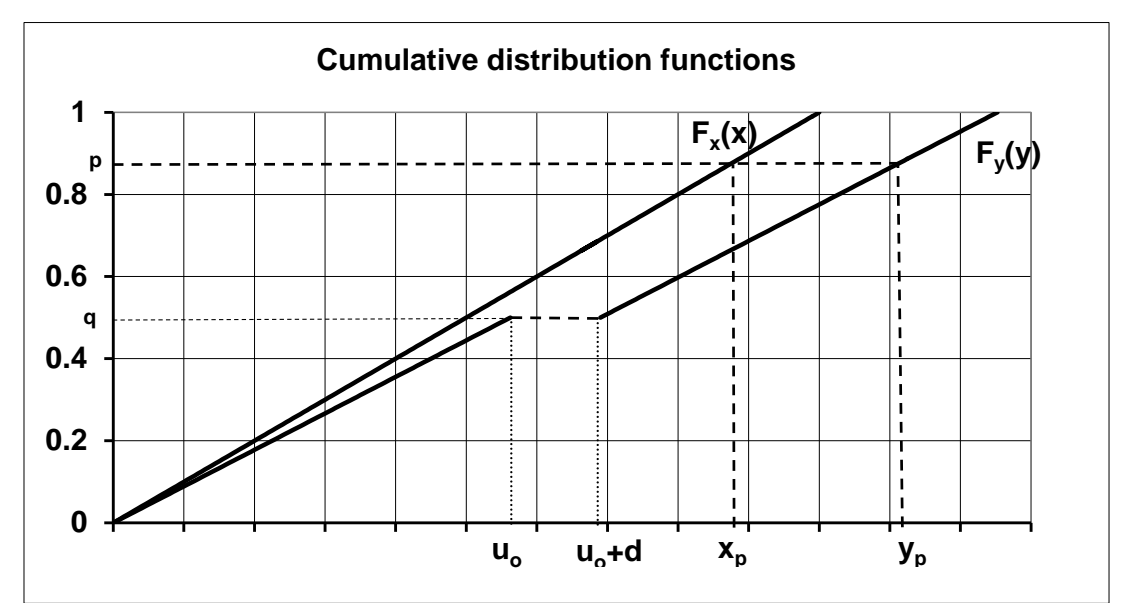

Figure 4. Sketch of cumulative distribution function for $X$ and $Y=h(X)$. Note the stochastic dominance $y_{p}>x_{p}$ for all $p$ and the jump in the distribution function $\bar{F}_{Y}(y)$ for $q$.

Now, the transformation $\bar{h}(x)$ is continuous for all $\delta>0$, and $y_{q-\delta}=\left(\mu_{X}+\rho\right) \bar{L}^{\prime}\left(F_{X}\left(x_{q-\delta}\right)\right) \geq x_{q-\delta}$. When $\delta \rightarrow 0_{+}$, the inequality holds for the limits and we obtain $y_{q_{-}} \geq x_{q}$. Similarly, we obtain $y_{q+\delta}=\left(\mu_{X}+\rho\right) \bar{L}^{\prime}\left(F_{X}\left(x_{q+\delta}\right)\right) \geq x_{q+\delta}$ and, when $\delta \rightarrow 0_{+}$, the inequality holds for the limits and we obtain $y_{q^{+}} \geq x_{q}$. Hence, $y_{p} \geq x_{p}$ for all $p$, and $Y=\bar{h}(X)$ stochastically dominates the initial variable $X$.

\section{Discussion}

We have studied the effects of transfer policies in this paper. In general, a transformation describing a realistic transfer policy has to be continuous. However, the theory presented is obviously applicable in connection with 
other income redistributive studies such that the discontinuity cannot be excluded. If the problem is reductions in taxation, then the tax reduction for a taxpayer can be considered as a new benefit [7]. The class of transfer policies $\boldsymbol{H}^{*}$ can consequently be used for comparisons between different tax-reducing policies. If changes of transfers are of interest, then the transfer policies can also be applied in transfer-raising situations. If transfers are increased, the effect of increases on a receiver can be considered through transfer policies belonging to $\boldsymbol{H}^{\boldsymbol{*}}$. In general, the changes may be mixtures of several different components and discontinuity cannot be excluded. The continuity assumption can be dropped and the class $\boldsymbol{H}^{*}$ of transfer policies containing discontinuous policies satisfies the same properties as the initial class discussed in ([5] [6]). Analogously, tax increases and transfer reductions can be considered as new tax policies [7]. One main result is still that continuity is a necessary condition if one pursues the notion that income inequality should remain or be reduced.

Empirical applications of the optimal policies among a class of tax policies and the class of transfer policies considered here have been discussed in ([2] [9]), where we developed "optimal yardsticks" to gauge the effectiveness of given real tax and transfer policies in reducing inequality.

\section{Conclusion}

We have studied the effects of discontinuous transfer policies. The theory presented is applicable in connection with income redistributive studies such that the discontinuity cannot be excluded. A tax reduction for a taxpayer or a transfer increase on a receiver can be considered as new benefits. In general, such changes may be mixtures of different policy components and discontinuity cannot be excluded. However, one main result is still that continuity is a necessary condition if income inequality should remain or be reduced.

\section{Acknowledgements}

This work was supported in part by a grant from the Magnus Ehrnrooths Stiftelse foundation.

\section{References}

[1] Fellman, J. (2013) Properties of Non-Differentiable Tax Policies. Theoretical Economics Letters, 3, 142-145. http://dx.doi.org/10.4236/tel.2013.33022

[2] Fellman, J. (2014) Mathematical Analysis of Distribution and Redistribution of Income. Science Publishing Group, 166 p. http://www.sciencepublishinggroup.com/book/B-978-1-940366-25-8.aspx

[3] Jakobsson, U. (1976) On the Measurement of the Degree of Progression. Journal of Public Economics, 5, $161-169$. http://dx.doi.org/10.1016/0047-2727(76)90066-9

[4] Fellman, J. (2009) Discontinuous Transformations, Lorenz Curves and Transfer Policies. Social Choice and Welfare, 33, 335-342. http://dx.doi.org/10.1007/s00355-008-0362-4

[5] Fellman, J. (1980) Transformations and Lorenz Curve. Swedish School of Economics and Business Administration Working Paper 49, 19 p.

[6] Fellman, J. (2003) On Lorenz Curves Generated by a Given Class of Transfer Policies. In: Höglund, R., Jäntti, M. and Rosenqvist, G., Eds., Statistics, Econometrics and Society: Essays in Honour of Leif Nordberg, Statistics Finland, Research Reports Number 239, 27-40.

[7] Fellman, J. (2001) Mathematical Properties of Classes of Income Redistributive Policies. European Journal of Political Economy, 17, 195-209. http://dx.doi.org/10.1016/S0176-2680(00)00035-5

[8] Fellman, J. (1995) Intrinsic Mathematical Properties of Classes of Income Redistributive Policies. Working Papers, No. 306, Hanken School of Economics, Helsinki, 26 p.

[9] Fellman, J., Jäntti, M. and Lambert, P.J. (1999) Optimal Tax-Transfer Systems and Redistributive Policy. Scandinavian Journal of Economics, 101, 115-126. http://dx.doi.org/10.1111/1467-9442.00144 